05,12

\title{
Энергия вытянутой сфероидальной оболочки в однородном магнитном поле
}

\author{
(C) Ю.А. Кокшаров \\ Московский государственный университет им. М.В. Ломоносова, \\ Москва, Россия \\ Институт общей и неорганической химии им. Н.С. Курнакова РАН, \\ Москва, Россия \\ Институт радиотехники и электроники им. В.А. Котельникова РАН, \\ Москва, Россия \\ E-mail: yak@physics.msu.ru
}

(Поступила в Редакцию 6 июля 2016 г.)

\begin{abstract}
Задача об энергии сфероидальной магнитной оболочки, решаемая методами классической электродинамики, возникает, в частности, при исследовании тонкостенных биосовместимых микрокапсул с целью решения актуальной проблемы - направленной доставки лекарств. Лекарство внутри микрокапсул должно быть освобождено в нужный момент посредством разрушения оболочки капсулы. Размещение в оболочке микрокапсул магнитных наночастиц, чувствительных к внешнему магнитному полю, позволяет в принципе решить обе задачи - доставить капсулу в нужное место и разрушить ее оболочку. Разрушение оболочки может, в частности, произойти под действием внутренних напряжений при изменении формы капсулы. Проведен анализ модели магнитной микрокапсулы в виде вытянутой сфероидальной магнитной оболочки, получены формулы для магнитостатической и магнитной свободной энергии в случае направления магнитного поля вдоль длинной оси сфероида.
\end{abstract}

Работа выполнена при поддержке РНФ (проект 14-12-01379).

DOI: 10.21883/FTT.2017.04.44271.283

\section{1. Введение}

Свойства магнитных оболочек во внешнем магнитном поле долгое время изучались в связи с задачей магнитного экранирования [1,2]. Задача об экранирующей магнитной оболочке сферической формы хорошо изучена [3]. В геофизических задачах магнитного детектирования исследуются объекты, моделируемые оболочками эллипсоидальной формы [4]. Эллипсоидальную форму могут иметь также микрокапсулы, липосомы и подобные микро- и наноструктуры, активно изучаемые в рамках проблемы направленной доставки лекарств [5]. В этой связи возникает задача применения внешнего электромагнитного поля для контролируемого разрушения (активации) микрокапсулы - носителя лекарств [6]. Чтобы использовать магнитное поле, в оболочку (мембрану) капсулы встраиваются биосовместимые (на основе оксидов железа) магнитные наночастицы [7]. Для такой микрокапсулы применима модель магнитной оболочки, толщина которой существенно меньше размера капсулы. Локализованные в оболочке магнитные наночастицы оксидов железа, как правило, суперпарамагнитны при комнатной температуре [8], и в небольших магнитных полях (менее $\approx 1$ кОе, [9]) оболочка капсулы может характеризоваться постоянной магнитной проницаемостью. В линейном приближении скалярный потенциал сфероидальной оболочки, намагничиваемой однородным внешним полем, найден в [10]. Цель настоящей работы - используя соотношения, полученные в [10], получить и проанализировать формулы для магнитостатической и свободной энергии вытянутой сфероидальной оболочки. Особый интерес представляет понижение магнитной энергии при изменении формы оболочки, что должно указывать на возможность спонтанной деформации реальных микрокапсул и, как следствие, возникновения в ней сильных механических деформаций, необходимых для разрушения. В этом отношении наша задача родственна классической проблеме электростатической неустойчивости Рэлея-Тейлора $[11,12]$, в частности, задаче об устойчивости заряженного эллипсоида [13].

\section{2. Параметры модели вытянутой сфероидальной магнитной оболочки}

Ограничимся рассмотрением вытянутой сфероидальной оболочки во внешнем магнитном поле, направленном вдоль длинной главной оси. Электростатическая аналогия [11] подсказывает, что сплюснутый магнитный сфероид обладает большей энергией, чем вытянутый того же объема. В магнитном поле вытянутый сфероид должен ориентироваться длинной осью вдоль внешнего поля [14].

На рис. 1 показано схематическое изображение сфероидальной оболочки, состоящей из двух софокусных сфероидов. Внешний (внутренний) сфероид характеризуется длинной полуосью $c_{e}\left(c_{i}\right)$ и короткой $b_{e}\left(b_{i}\right)$. Уравнения 


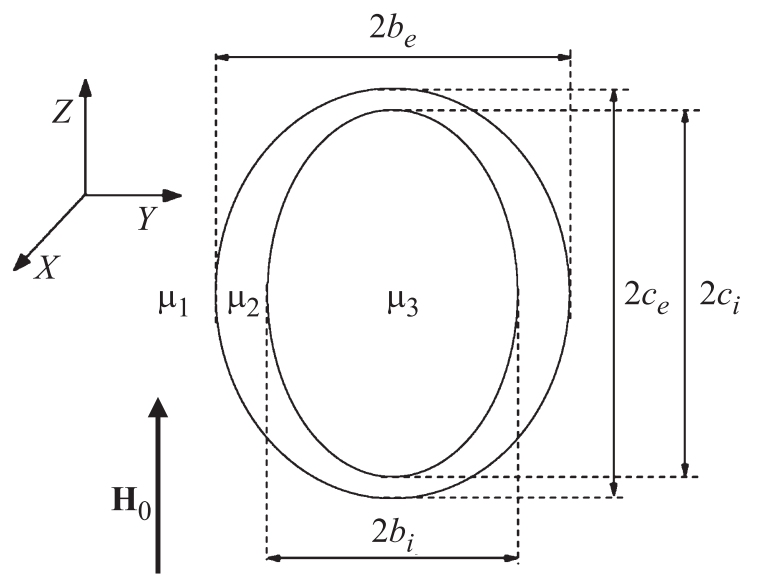

Рис. 1. Модель магнитной сфероидальной оболочки. Декартовые координаты $(x, y, z)$ поверхностей заданы уравнением (1). Формулы (5), (6) задают связь длин полуосей $c_{i, e}$ и $b_{e, i}$ с параметром $\eta$. Магнитные проницаемости внешнего пространства, материала оболочки и ее внутреннего пространства (полости) обозначены $\mu_{1}, \mu_{2}$ и $\mu_{3}$ соответственно.

сфероидов в декартовых координатах:

$$
x^{2} /\left(b_{e, i}^{2}+\lambda\right)+y^{2} /\left(b_{e, i}^{2} 2+\lambda\right)+z^{2} /\left(c_{e, i}^{2}+\lambda\right)=1,
$$

где $\lambda$ - параметр семейства софокусных сфероидов.

Для нахождения магнитного скалярного потенциала $V$, связь которого с напряженностью магнитного поля $\mathbf{H}$ дается формулой

$$
H=-\operatorname{grad}(V),
$$

удобно ввести вытянутые сфероидальные координаты (ВСК) $\eta$ и $\xi$, связанные с декартовыми координатами соотношениями [10]

$$
\begin{gathered}
z=f \eta \xi, \\
x^{2}+y^{2}=f\left[\left(\eta^{2}-1\right)\left(1-\xi^{2}\right)\right]^{1 / 2},
\end{gathered}
$$

где $f=\left(c^{2}-b^{2}\right)^{1 / 2}-$ половина фокусного расстояния сфероида. Область изменения введенных таким образом координат: $1 \leq \eta<\infty,-1 \leq \xi \leq+1$. Третьей координатой в системе ВСК является угол $\varphi$ сферической системы координат. Связь $\eta$ и $\xi$ с длинами полуосей дается формулами

$$
\begin{gathered}
c=f \eta, \\
b=f\left(\eta^{2}-1\right)^{1 / 2} .
\end{gathered}
$$

Для точек, лежащих на поверхности вытянутого сфероида, $\eta=$ const. Внешняя и внутренняя поверхности сфероидальной оболочки задаются параметрами $\eta_{e}$ и $\eta_{i}$ соответственно. Объем сфероидального слоя $\Omega_{\mathrm{sh}}$, ограниченного такими поверхностями, равен

$$
\Omega_{\mathrm{sh}}=(4 / 3) \pi f^{3}\left\{\eta_{e}\left(\eta_{e}^{2}-1\right)-\eta_{i}\left(\eta_{i}^{2}-1\right)\right\} .
$$

Площадь поверхности вытянутого сфероида равна

$$
S=2 \pi f^{2}\left(\eta^{2}-1\right)^{1 / 2}\left[\eta^{2} \arcsin (1 / \eta)+\left(\eta^{2}-1\right)^{1 / 2}\right] .
$$

Эксцентриситет $e$ вытянутого сфероида обратно пропорционален $\eta^{2}$

$$
e=\left(c^{2}-b^{2}\right) / c^{2}=1 / \eta^{2} .
$$

Магнитные параметры модели включают магнитные проницаемости: внешнего пространства $-\mu_{1}$, вещества оболочки $-\mu_{2}$ и внутреннего пространства (полости) $-\mu_{3}$.

Моделирование реальной оболочки микрокапсулы сфероидальным слоем имеет недостаток - толщина оболочки неодинакова в разных точках поверхности капсулы (рис. 1). Однако такой выбор позволяет получить достаточно простое аналитическое выражение для электро- или магнитостатического потенциала и поэтому удобен для теоретического анализа $([10,15,16])$.

\section{3. Энергия сфероидальной вытянутой оболочки в однородном магнитном поле, направленном вдоль длинной главной оси}

Задача о поляризуемой в однородном внешнем поле эллипсоидальной магнитной или диэлектрической линейной оболочке может быть решена точно с помощью уравнения Лапласа. Наиболее общее решение электростатической задачи приведено в [16]. Однако полученные в [16] формулы для потенциала содержат неаналитические функции, что затрудняет их использование для расчета энергии. Для частного случая сфероидальной магнитной оболочки (рис. 1) получаются относительно простые формулы для потенциала в области вне оболочки $\left(V_{1}\right)$ и во внутренней полости $\left(V_{3}\right)$. Для вытянутого сфероида, ориентированного длинной осью вдоль однородного внешнего магнитного поля $\mathbf{H}_{0}$, они имеют вид [10] (в системе ВСК)

$$
\begin{gathered}
V_{1}(\eta, \xi)=\eta \xi \mathbf{H}_{0}\left(-f+K(\eta) \Delta_{1} / \Delta_{0}\right), \\
V_{3}(\eta, \xi)=\eta \xi \mathbf{H}_{0} \Delta_{3} / \Delta_{0},
\end{gathered}
$$

где $K(\eta)=0.5 \ln [(\eta+1) /(\eta-1)]-1 / \eta$. Величины $\Delta_{0}$, $\Delta_{1}, \Delta_{3}$ представлены в [10] в виде детерминантов матриц $4 \times 4$, в качестве элементов содержащих комбинации постоянных - параметров модели. Для расчета энергии в нашей работе использовались явные выражения (12)-(14) для $\Delta_{0}, \Delta_{1}, \Delta_{3}$, полученные раскрытием упомянутых детерминантов

$$
\begin{aligned}
& \Delta_{0}=-K^{2}\left(\eta_{e}\right)\left(\mu_{1}-\mu_{2}\right)\left(\mu_{2}-\mu_{3}\right) \\
& +\mu_{1} \eta_{e} k\left(\eta_{e}\right)\left(K\left(\eta_{i}\right)\left(\mu_{2}-\mu_{3}\right)+\mu_{2} \eta_{i} k\left(\eta_{i}\right)\right) \\
& +K\left(\eta_{e}\right)\left(\mu_{1}-\mu_{2}\right)\left(\left(K\left(\eta_{i}\right)-\eta_{e} k_{e}\right)\left(\mu_{2}-\mu_{3}\right)+\mu_{2} \eta_{i} k\left(\eta_{i}\right)\right), \\
& \Delta_{1}=-f\left[\left(\mu_{1}-\mu_{2}\right)\left(\mu_{2}-\mu_{3}\right)\left\{K\left(\eta_{e}\right) K\left(\eta_{i}\right)\right\}\right. \\
& \left.\quad+\mu_{2}\left\{\eta_{e} k_{e}\left(\mu_{3}-\mu_{2}\right)+\eta_{i} k\left(\eta_{i}\right)\left(\mu_{2}-\mu_{1}\right)\right\}\right], \\
& \quad \Delta_{3}=-f \mu_{1} \mu_{2} \eta_{e} k\left(\eta_{e}\right) \eta_{i} k\left(\eta_{i}\right),
\end{aligned}
$$


где

$$
k(\eta) \equiv d K(\eta) / d \eta=1 /\left(\eta^{2}-\eta^{4}\right)
$$

Отметим ошибку в работе [10] — во всех формулах, содержащих величину $f$, следует заменить ее на ,- $f^{“}$, чтобы обеспечить убывание потенциала в направлении внешнего магнитного поля.

Вопрос расчета энергии намагниченного тела во внешнем поле не является тривиальным [17-19]. Сначала мы получим формулу для магнитостатической энергии, которая для линейного магнетика равна поверхностному интегралу от произведения магнитного фиктивного заряда на потенциал [20]. Для оболочки интегрирование производится по внешней и внутренней поверхности

$$
W=\frac{\mu_{0}}{2} \int_{\text {surf }} \sigma V d S=\frac{\mu_{0}}{2} \int_{\text {ext }} \sigma_{e} V_{e} d S+\frac{\mu_{0}}{2} \int_{\text {int }} \sigma_{i} V_{i} d S,
$$

где $\sigma_{e}$ и $\sigma_{i}-$ плотность фиктивных магнитных зарядов $\sigma$ на внешней и внутренней поверхностях оболочки соответственно. Вектор элемента площади $d \mathbf{S}$ выражается формулой [21]

$$
d \mathbf{S}=f^{2}\left[\left(\eta^{2}-1\right)\left(\eta^{2}-\xi^{2}\right)\right]^{1 / 2} d \xi d \varphi \boldsymbol{\eta}
$$

где $\varphi-$ азимутальный угол сферической системы координат, $\boldsymbol{\eta}$ - единичная нормаль к поверхности $\eta=$ const. В силу аксиальной симметрии интегрирование (16) по углу $\varphi$ дает множитель $2 \pi$. Таким образом, вычисление энергии $W$ в (16) сводится к интегрированию по переменной $\xi$ в пределах от -1 до +1 .

Из (10) и (11) следует, что потенциал поверхностей оболочки в силу его непрерывности равен соответственно

$$
V_{e}=V_{1}\left(\eta_{e}, \xi\right)=\eta_{e} \xi \mathbf{H}_{0}\left(-f+K\left(\eta_{e}\right) \Delta_{1} / \Delta_{0}\right)
$$

и

$$
V_{i}=V_{3}\left(\eta_{i}, \xi\right)=\eta_{i} \xi \mathbf{H}_{0} \Delta_{3} / \Delta_{0}
$$

Плотность фиктивных магнитных зарядов можно найти, используя граничные условия (20), (21) и материальные уравнения (22), (23)

$$
\begin{aligned}
& H_{1 n}-H_{2 n}=\sigma_{e} \\
& H_{2 n}-H_{3 n}=\sigma_{i} \\
& \mu_{1} H_{1 n}=\mu_{2} H_{2 n} \\
& \mu_{2} H_{2 n}=\mu_{3} H_{3 n} .
\end{aligned}
$$

Нормальная компонента напряженности находится с помощью соотношения (24), вытекающего из (2)

$$
H_{n}=-\partial V / \partial n=-(1 / f)(\partial V / \partial \eta)\left[\left(\eta^{2}-1\right) /\left(\eta^{2}-\xi^{2}\right)\right]^{1 / 2},
$$

где единичный вектор нормали $\mathbf{n}$ направлен наружу от сфероида. Окончательные выражения для $\sigma_{e}$ и $\sigma_{i}$ имеют вид

$$
\begin{aligned}
\sigma_{e}= & -(1 / f)\left(\partial V_{1} / \partial \eta\right)\left[\left(\eta_{e}^{2}-1\right) /\left(\eta_{e}^{2}-\xi^{2}\right)\right]^{1 / 2}\left(1-\mu_{1} / \mu_{2}\right) \\
= & \xi H_{0}(1 / f)\left[f-\left\{K\left(\eta_{e}\right)+\eta_{e} k\left(\eta_{e}\right)\right\} \Delta_{1} / \Delta_{0}\right] \\
& \times\left[\left(\eta_{e}^{2}-1\right) /\left(\eta_{e}^{2}-\xi^{2}\right)\right]^{1 / 2}\left(1-\mu_{1} / \mu_{2}\right) \\
\sigma_{i}= & -(1 / f)\left(\partial V_{3} / \partial \eta\right)\left[\left(\eta_{i}^{2}-1\right) /\left(\eta_{i}^{2}-\xi^{2}\right)\right]^{1 / 2}\left(\mu_{3} / \mu_{2}-1\right) \\
= & \xi H_{0}(1 / f)\left(\Delta_{3} / \Delta_{0}\right)\left[\left(\eta_{i}^{2}-1\right) /\left(\eta_{i}^{2}-\xi^{2}\right)\right]^{1 / 2}\left(1-\mu_{3} / \mu_{2}\right) .
\end{aligned}
$$

После подстановки (18), (19), (25), (26) в (16) и интегрирования выражение для магнитостатической энергии принимает вид

$$
\begin{aligned}
W & =\mu_{0}\left\{\left(2 \pi f H_{0}^{2} / 3\right) \eta_{i}\left(\Delta_{3} / \Delta_{0}\right)^{2}\left(\eta_{i}^{2}-1\right)\left(1-\mu_{3} / \mu_{2}\right)\right. \\
& +\left(2 \pi f^{2} H_{0}^{2} / 3\right) \eta_{e}\left(-f+K\left(\eta_{e}\right) \Delta_{1} / \Delta_{0}\right) \\
& \left.\times\left[f-\left\{K\left(\eta_{e}\right)+\eta_{e} k\left(\eta_{e}\right)\right\} \Delta_{1} / \Delta_{0}\right]\left(\eta_{e}^{2}-1\right)\left(1-\mu_{1} / \mu_{2}\right)\right\} .
\end{aligned}
$$

Магнитостатическая энергия (27), согласно методу ее получения, включает энергию взаимодействия оболочки с внешним полем и собственную энергию (энергию размагничивания) [22]. В предельном случае $\eta_{i} \rightarrow 1$ и $\eta_{e} \rightarrow \infty$ (оболочка переходит в шар) выражение (27) принимает вид

$$
W=-(1 / 2) \mu_{0} m \mathbf{H}_{0}+(1 / 2) \mu_{0} N M^{2} \Omega_{\mathrm{sp}},
$$

где $N=1 / 3$ - размагничивающий фактор для шара, $\Omega_{\mathrm{sp}}$ - объем шара, $m=M \Omega_{\mathrm{sp}}$ - эффективный дипольный момент оболочки [10]

$$
m=4 \pi f^{2} \mathbf{H}_{0}\left(\Delta_{1} / 3 \Delta_{0}\right),
$$

определяющий ее магнитный потенциал на больших расстояниях, М - намагниченность. Первое слагаемое в (28) равно работе внешних сил по переносу отдельных тонких „стержней“ (с $N=0)$, на которые можно мысленно разбить шар, из области с нулевым магнитным полем в точку поля с напряженностью $\mathbf{H}_{0}$. Второе слагаемое в (28) равно работе внешних сил по „сборке“ этих стержней (с „замороженной“ намагниченностью) в единое тело в форме шара [23].

При $\mu_{1}=\mu_{3}=1$ и предельном переходе $\eta_{i} \rightarrow 1$, $\eta_{e} \rightarrow \infty$ из (29) получается известный результат для намагниченности шара из линейного магнетика в однородном внешнем поле (в вакууме)

$$
M=m / V=3 \mathbf{H}_{0}\left\{\left(\mu_{2}-1\right) /\left(\mu_{2}+2\right)\right\} .
$$

Подстановка (30) в (28) показывает, что магнитостатическая энергия намагниченного шара стремится к нулю при $\mu_{2} \rightarrow \infty$. Численный расчет по формуле (27) подтверждает эту закономерность для сфероидальных оболочек произвольной толщины и эксцентриситета. На 


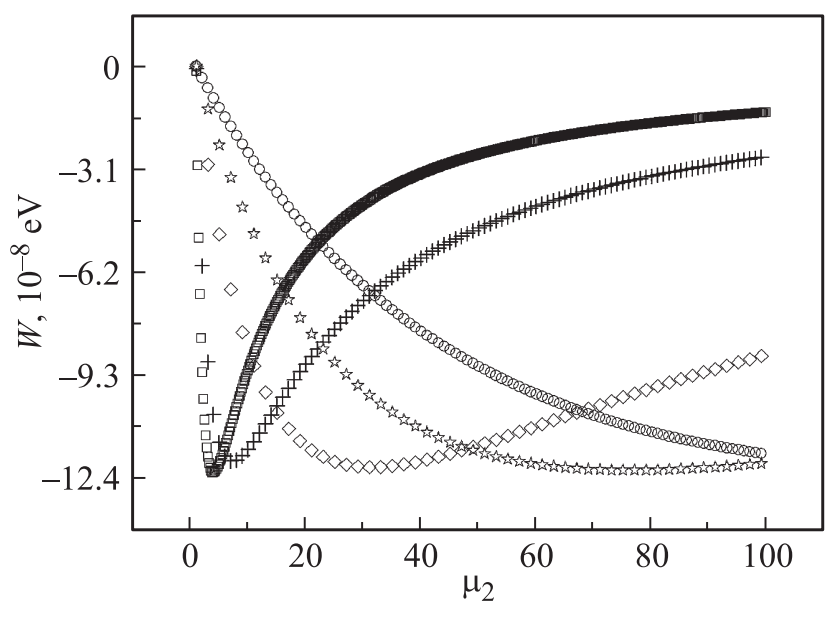

Рис. 2. Зависимость магнитостатической энергии (27) от магнитной проницаемости $\mu_{2}$ для оболочек различной толщины. Внешняя поверхность оболочки близка к сферической: $\eta_{e}=100$. Значения $\eta_{i}$ : квадраты $-0.01 \eta_{e}$; крестики $-0.75 \eta_{e}$; ромбы $-0.95 \eta_{e}$; звездочки $-0.98 \eta_{e}$; кружки $-0.99 \eta_{e}$. Квадраты описывают зависимость (28) для намагниченного шара. Другие параметры расчета: $f=1 \mu \mathrm{m}, H_{0}=1 \mathrm{Oe}, \mu_{1}=\mu_{3}=1$.

рис. 2 показаны графики зависимости магнитостатической энергии от магнитной проницаемости $\mu_{2}$ для оболочек различной толщины (для $\mu_{1}=\mu_{3}=1$ ). Стремление $W$ к нулю при $\mu_{2} \rightarrow \infty$ кажется абсурдным очевидно, что при внесении намагничиваемого тела в магнитное поле нужно совершить работу, однако изменение магнитной энергии при этом оказывается равным нулю. Для разрешения этого кажущегося противоречия полезно обратиться к электростатической аналогии. Вопервых, предел $\mu \rightarrow \infty$ соответствует пределу $\varepsilon \rightarrow \infty$, где $\mu$ и $\varepsilon-$ магнитная и диэлектрическая проницаемость тела соответственно. Во-вторых, предел $\varepsilon \rightarrow \infty$ соответствует формальному переходу от диэлектрика к проводнику. Проводник эквипотенциален, поэтому расчет энергии по формуле, аналогичной (16), даст нулевое значение энергии (полный заряд тела равен нулю). Поэтому и при внесении в постоянное электрическое поле проводника изменение вклада (16) в полную энергию равно нулю. Однако закон сохранения энергии требует учесть работу, необходимую для поддержания постоянного внешнего поля. Она совершается над зарядами источниками постоянного поля, и равна (со знаком ,-““) работе по внесению тела в постоянное электрическое (магнитное) поле. Таким образом, для линейного магнетика в постоянном внешнем поле магнитостатическая энергия $W$, рассчитанная по формуле (16), не определяет работу механических внешних сил при перемещении или деформации магнетика.

Для расчета силы и момента силы, действующей на намагниченное тело, необходимо, в общем случае, использовать не магнитостатическую энергию $W$, а свободную энергию $F$ [19]. Рассмотрим случай $\mu_{1}=\mu_{3}=1$. Воспользуемся формулой из работы [24] для свободной энергии тела с проницаемостью $\mu$, намагничиваемого в постоянном магнитном поле $\mathbf{H}_{0}$

$$
F=-\frac{\mu_{0}(\mu-1)}{2} \int_{V} \mathbf{H}_{1} \mathbf{H}_{0} d \Omega,
$$

где $\mathbf{H}_{1}$ полная напряженность внутри тела. Отметим, что так как

$$
\mathbf{M}=(\mu-1) \mathbf{H}_{1},
$$

свободная энергия (31) равна изменению магнитной энергии, представляемой в виде

$$
W_{\text {magn }}=\int \frac{\mathbf{B H}}{2} d \Omega
$$

при внесении линейного магнетика в „фиксированное“ внешнее поле (например, создаваемого постоянными магнитами) [25]

$$
\Delta W_{\text {magn }}=-\frac{\mu_{0}}{2} \int_{V} \mathbf{M H}_{0} d \Omega .
$$

С учетом ранее введенных обозначений (31) преобразуется к виду

$$
\begin{aligned}
F & =\frac{\mu_{0}\left(\mu_{2}-1\right)}{2} \int_{V} \nabla V_{2} \mathbf{H}_{0} d \Omega \\
& =\frac{\mu_{0}\left(\mu_{2}-1\right) \mathbf{H}_{0}}{2} \int_{V} \nabla V_{2} d \Omega=\frac{\mu_{0}\left(\mu_{2}-1\right) \mathbf{H}_{0}}{2} \int_{S} V_{2} d S_{z} \\
& =\frac{\mu_{0}\left(\mu_{2}-1\right) H_{0}}{2}\left(\int_{\text {ext }} V_{1} d S_{z}+\int_{\text {int }} V_{3} d S_{z}\right)
\end{aligned}
$$

где мы использовали непрерывность потенциала на поверхности оболочки и формулу преобразования объемного интеграла градиента:

$$
\int_{\Omega} \nabla V d \Omega=\int_{S} V d \mathbf{S} .
$$

Для нахождения величины $d S_{z}$, используемой в (35), спроецируем вектор внешней нормали $d \mathbf{S}$ к поверхности $\eta=$ const (см. (17)) на направление внешнего поля. Для этого воспользуемся формулой разложения коллинеарного ему единичного вектора $\boldsymbol{\eta}$ на декартовые оси [21]

$$
\begin{aligned}
\boldsymbol{\eta}= & \eta\left[\left(1-\xi^{2}\right) /\left(\eta^{2}-\xi^{2}\right)\right]^{1 / 2}(\mathbf{i} \cos \varphi+\mathbf{j} \sin \varphi) \\
& +\xi\left[\left(\eta^{2}-1\right) /\left(\eta^{2}-\xi^{2}\right)\right]^{1 / 2} \mathbf{k}
\end{aligned}
$$

где $\mathbf{i}, \mathbf{j}, \mathbf{k}$ - орты декартовой системы координат. Таким образом,

$$
d S_{z}= \pm \xi f^{2}\left(\eta^{2}-1\right) d \xi d \varphi
$$

где знак „+““ применяется для внешней поверхности оболочки, „-““- для внутренней. Используя (18), (19), (38), 
после алгебраических преобразований и интегрирования в (35) для свободной энергии имеем

$$
\begin{aligned}
& F=\mu_{0}\left(\mu_{2}-1\right)\left(2 \pi f^{2} H_{0}^{2} / 3\right) \\
& \quad \times\left\{\left(\eta_{e}^{2}-1\right) \eta_{e}\left(-f+K\left(\eta_{e}\right) \Delta_{1} / \Delta_{0}\right)-\left(\eta_{i}^{2}-1\right) \eta_{i} \Delta_{3} / \Delta_{0}\right\} .
\end{aligned}
$$

При $\eta_{i} \rightarrow 1$ и $\eta_{e} \rightarrow \infty$ (случай шара) выражение (39) переходит в

$$
F=-(3 / 2) \mu_{0} H_{0}^{2}\left\{\left(\mu_{2}-1\right) /\left(\mu_{2}+2\right)\right\} \Omega_{\mathrm{sp}},
$$

где $\Omega_{\mathrm{sp}}=(4 \pi / 3) f^{3} \eta_{e}^{3}$ - объем шара.

В рамках рассматриваемой модели свободная энергия (39) определяет изотермическую работу внешних сил при перемещении или деформации (сохраняющей софокусность) намагничиваемой оболочки.

\section{4. Зависимость свободной энергии сфероидальной магнитной оболочки от ее формы}

Как следует из (39), свободная энергия сфероидальной оболочки должна зависеть от ее формы. Для тонкой оболочки $\eta_{e} \approx \eta_{i}$ и, согласно (9), форму оболочки можно описывать одним параметром - эксцентриситетом $e \approx 1 / \eta_{e}^{2} \approx 1 / \eta_{i}^{2}$. Для толстой оболочки (хотя такой случай не реализуется для интересующих нас микрокапсул) форму оболочки можно характеризовать двумя параметрами, например эксцентриситетом внешней поверхности и отношением $\eta_{i} / \eta_{e}$.

Рассмотрим изменение формы вытянутой сфероидальной оболочки при деформации, сохраняющей полный объем, объем полости, а следовательно, и объем оболочки (пространства между сфероидами). Это соответствует несжимаемости водной среды в полости микрокапсулы, а также материала ее оболочки. Для применимости формул, полученных в предыдущем разделе, потребуем, чтобы после деформации сфероидальные поверхности оставались софокусными. Тогда должны выполняться следующие соотношения:

$$
\begin{gathered}
f_{1}^{3} \eta_{e 1}\left(\eta_{e 1}^{2}-1\right)=f_{2}^{3} \eta_{e 2}\left(\eta_{e 2}^{2}-1\right), \\
f_{1}^{3} \eta_{i 1}\left(\eta_{i 1}^{2}-1\right)=f_{2}^{3} \eta_{i 2}\left(\eta_{i 2}^{2}-1\right)
\end{gathered}
$$

Индексы 1 и 2 указывают на состояния до и после деформации соответственно. Опишем процедуру получения зависимости свободной энергии от формы оболочки. Сначала для фиксированных начальных значений $\eta_{e 1}$ и $\eta_{i 1}<\eta_{e 1}$ вычисляется $F$ по формуле (39). Начальная форма оболочки такова, что внешняя поверхность почти сферическая, для этого выбирается $\eta_{e 1} \gg 1\left(\eta_{e 1}=100\right.$ для данных, приведенных на рис. 2 и рис. 3 ). Начальное значение межфокусного расстояния должно быть мало (так как для сферы $f=0$ ), можно выбрать $f_{1}=1 / \eta_{e 1}<<1$. После вычисления $F$ для начальных параметров значение $f$ немного увеличивается:

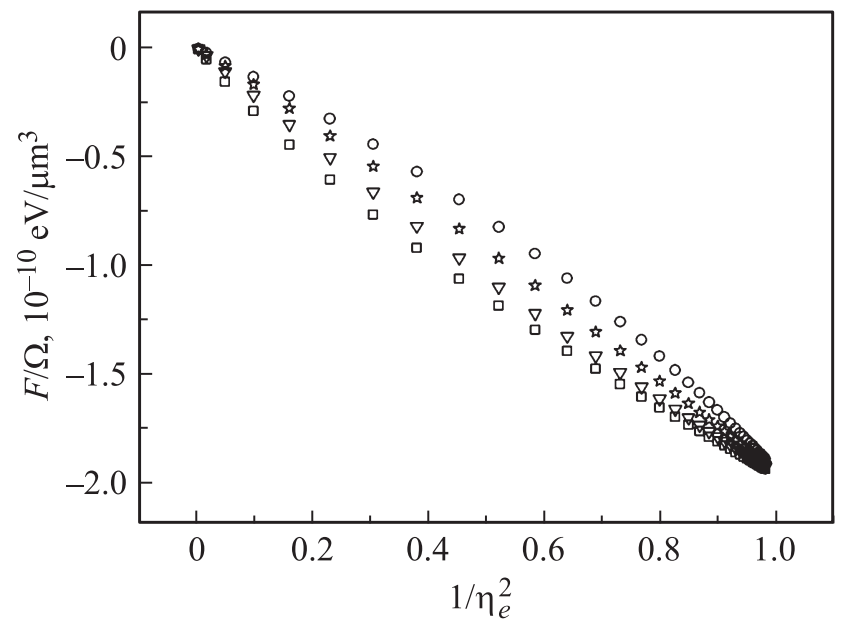

Рис. 3. Зависимость объемной плотности свободной магнитной энергии оболочки (39) от эксцентриситета внешней поверхности $e=1 / \eta_{e}^{2}$ для различных значений толщины оболочки (отношения $\eta_{i} / \eta_{e}$ ). Минимальный эксцентриситет соответствует начальным значениям параметров: $\eta_{e}=100$ и $\eta_{i}=0.99 \eta_{e}$ (кружки), $0.75 \eta_{e}$ (звездочки), $0.5 \eta_{e}$ (треугольники), $0.01 \eta_{e}$ (квадраты). Для квадратов свободная энергия описывается формулой (40). При изменении эксцентриситета объем оболочки сохраняется. Другие параметры расчета: $H_{0}=1 \mathrm{Oe}$, $\mu_{1}=\mu_{3}=1 ; \mu_{2}=1.01$.

$f_{2}=f_{1}+0.01$. Шаг изменения $f$ определяется общим числом точек получаемой зависимости и потенциальной возможностью численного дифференцирования. Далее следует найти $\eta_{e 2}$ и $\eta_{i 2}$, удовлетворяющие (41) и (42), с помощью следующего алгоритма. Заданы $\eta_{e 1}, \eta_{i 1}$, $f_{1}$ и $f_{2}$. Уравнения (41) и (42) приводятся к двум аналогичным кубическим уравнениям (для $x=\eta_{e 2}$ и $\eta_{i 2}$ )

$$
x^{3}+p x+q=0
$$

где $\quad p=-1 ; \quad q=-\left(f_{1} / f_{2}\right)^{3} \eta_{(e, i) 1}\left(\eta_{(e, i) 1}^{2}-1\right)<0$. Решение (единственное, при условии $x>1$ ) находится по стандартным алгебраическим формулам. Если $|q|<2 /(27)^{1 / 2}, \quad x=(4 / 3)^{1 / 2} \sin (\alpha / 3+2 \pi / 3)$, где $\quad \alpha=(4 / 3)^{1 / 2} \arcsin \left\{(9 q / 4)(4 / 3)^{1 / 2}\right\} . \quad$ Если $|q|>2 /(27)^{1 / 2}, \quad x=\left(-0.5 q+\left(0.25 q^{2}-1 / 27\right)^{1 / 2}\right)^{1 / 3}$ $+\left(-0.5 q-\left(0.25 q^{2}-1 / 27\right)^{1 / 2}\right)^{1 / 3}$. Для полученных $\eta_{e 2}$, $\eta_{i 2}, f_{2}$ опять вычисляется $F$ с помощью (39). Далее описанная процедура выполняется, пока эксцентриситет не станет максимально возможным $(e \approx 1)$. Отметим, что при рассматриваемом типе деформации площадь поверхностей оболочки (8) практически не изменяется. Значения магнитной проницаемости оболочки $\mu_{2}$ для расчетов были выбраны в интервале $1.01-1.1$, учитывая невысокую концентрацию магнитных наночастиц в микрокапсулах и характерные значения 1.05-1.1 для феррожидкостей [26].

На рис. 3 показаны графики зависимости объемной плотности свободной энергии (39) от эксцентриситета внешней поверхности оболочки для различных значений толщины оболочки (значений $\eta_{i} / \eta_{e}$ ) при сохранении 


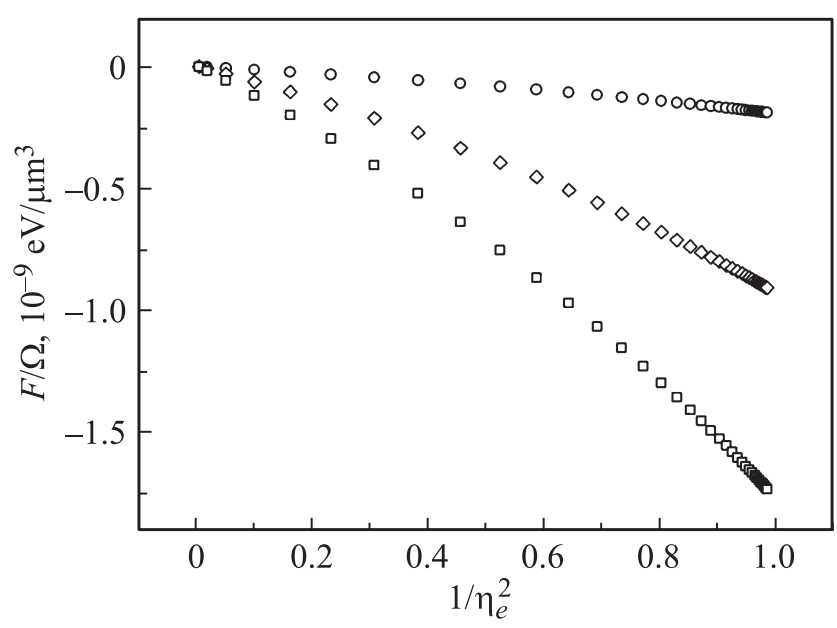

Рис. 4. Зависимость объемной плотности свободной магнитной энергии (39) тонкой оболочки от эксцентриситета внешней поверхности $e=1 / \eta_{e}^{2}$ при различных значениях магнитной проницаемости $\mu_{2}: 1.01$ (кружки), 1.05 (ромбы) и 1.1 (квадраты). Начальные значения параметров: $\eta_{2}=100$ и $\eta_{1}=0.99 \eta_{2}$. Объем оболочки сохраняется. Другие параметры расчета: $H_{0}=1 \mathrm{Oe}, \mu_{1}=\mu_{3}=1$.

объема оболочки. Видно, что свободная энергии уменьшается по мере вытягивания оболочки вдоль магнитного поля, причем чувствительность к изменению формы оболочки немного увеличивается с ростом толщины. На рис. 4 показана зависимость плотности свободной энергии от формы тонкой оболочки для разных значений $\mu_{2}$. При увеличении $\mu_{2}$ чувствительность $F$ к изменению формы оболочки заметно возрастает.

\section{5. Выводы}

Монотонное уменьшение магнитной свободной энергии по мере увеличения эксцентриситета сфероидальной вытянутой оболочки указывает на возможность неустойчивости такой оболочки, помещенной во внешнее магнитное поле. В отличие от электростатического проводящего эллипсоида увеличение поверхностного вклада в свободную энергию в процессе деформации не происходит. В реальных экспериментах устойчивость оболочки будет зависеть от упругих свойств как самой оболочки, так и окружающей ее среды. Возможность усиливать чувствительность магнитной свободной энергии к деформации, увеличивая магнитное поле или повышая магнитную проницаемость оболочки, позволяет надеяться на перспективность использования магнитных микрокапсул для решения проблемы адресной доставки лекарств.

\section{Список литературы}

[1] A.W. Rücker. Proc. Phys. Soc. London 12, 462, (1894).

[2] T. Omori, Y. Takeiri, B.J. Peterson. Jpn. J. Appl. Phys. 47, 3673 (2008).
[3] J.D. Jackson. Classical electrodynamics. John Willey \& Sons, N.Y. (1999). P. 201.

[4] J.E. McFee, Y. Das, R.O. Ellingson. IEEE Trans. Geosci. Rem. Sens. GE-28, 182 (1990).

[5] M. Bonini, D. Berti, P. Baglioni. Curr. Opin. Colloid \& Interface Sci. 18, 459 (2013).

[6] Ю.В. Гуляев, В.А. Черепенин, В.А. Вдовин, И.В. Таранов, В.В. Файкин, В.И. Тюкавин, В.П. Ким, Ю.А. Кокшаров, П.А. Кормакова, К.В. Потапенков, А.А. Рахнянская, А.В. Сыбачин, Е.Г. Ярославова, А.А. Ярославов, Г.Б. Хомутов. Журнал радиоэлектроники 14, 1 (2014).

[7] C. Bonnaud, C.A. Monnier, D. Demurtas, C. Jud, D. Vanhecke, X. Montet, R. Hovius, M. Lattuada, B. RothenRutishauser, A. Petri-Fink. ACS Nano 8, 3451 (2014).

[8] S. Laurent, D. Forge, M. Port, A. Roch, C. Robic, L.V. Elst, R.N. Muller. Chem.Rev. 108, 2064 (2008).

[9] С.Н. Ивичева, Ю.Ф. Каргин, Е.А. Овченков, Ю.А. Кокшаров, Г.Ю. Юрков. ФТТ 53, 1053 (2011).

[10] L. Frumkis, B.-Z. Kaplan. IEEE Trans. Magn. 35, 4151 (1999).

[11] Lord Rayleigh. Phil. Mag. 14, 184 (1882).

[12] G. Taylor. Proc. Roy. Soc. London. A 280, 383 (1964).

[13] С.И. Щукин, А.И. Григорьев. ФТТ 68, 48 (1997).

[14] A.R. Laufer. Am. J. Phys. 19, 275 (1951).

[15] K. Asami, T. Hanai, N. Koizumi. Jap. J. Apl. Phys. 19, 359 (1980).

[16] C.F. Bohren, D.R. Huffman. Absorption and Scattering of Light Small Particles. John Willey \& Sons, N.Y. (1983). P. 148.

[17] E.A. Guggenheim. Proc. Roy. Soc. A. 155, 49 (1936).

[18] E.A. Guggenheim. Proc. Roy. Soc. A. 155, 70 (1936).

[19] O. Narayan, A.P. Yang. Am. J. Phys. 73, 293 (2005).

[20] D.J. Craik. J. Phys. D. 7, 1566 (1974).

[21] F.M. Kahnert, J.J. Stamnes, K. Stamnes. J. Quant. Spectr. Rad. Transf. 77, 61 (2003).

[22] Magnetism. Fundamentals / Eds É. Du Trémolet de Lacheisserie, D. Gignoux, M. Schlenker. V. 1, Springer, Boston (2005). P. 61.

[23] M.S. Plesset, G. Venezian. Am. J. Phys. 32, 860 (1964).

[25] O.D. Jefimenko. Electricity and Magnetism. Appleton-Century-Crofts, N.Y. (1966). P. 488.

[26] М.И. Шлиомис. УФН 112, 427 (1974). 